\title{
Accuracy of BCS-based approximations for pairing in small Fermi systems
}

\author{
N. Sandulescu ${ }^{a, b}$ and G. F. Bertsch ${ }^{b}$ \\ ${ }^{a}$ Institute of Physics and Nuclear Engineering, 76900 Bucharest, Romania \\ ${ }^{b}$ DPTA/Service de Physique nucléaire, F-91680 Bruyères-le-Châtel, France \\ ${ }^{c}$ Institute of Nuclear Theory and Dept. of Physics, University of Washington, Seattle
}

\begin{abstract}
We analyze the accuracy of BCS-based approximations for calculating correlation energies and odd-even energy differences in 2-component fermionic systems with a small number of pairs. The analysis is focused on comparing BCS and projected BCS treatments with the exact solution of the pairing Hamiltonian, considering parameter ranges appropriate for nuclear pairing energies. We find that the projected BCS is quite accurate over the entire range of coupling strengths in spaces of up to about $\sim 20$ doubly degenerate orbitals. It is also quite accurate for two cases we considered with a more realistic Hamiltonian, representing the nuclei around ${ }^{117} \mathrm{Sn}$ and ${ }^{207} \mathrm{~Pb}$. However, the projected BCS significantly underestimates the energies for much larger spaces when the pairing is weak.
\end{abstract}

\section{INTRODUCTION}

A theory of nuclear pairing based on the BCS approximation was considered for the first time 50 years ago [1]. Since then, BCS-based approximations or more generally the Hartree-Fock-Bogoliubov equation have become ubiquitous for calculating nuclear energies in the framework of density functional theory or self-consistent mean-field theory. It is thus important to understand the limitations of these approximations and use more accurate theory when needed. In particular, the BCS Ansatz of a condensate with indefinite particle number becomes problematic in finite systems with weak pairing, as is the case for nuclei at shell closures. Several ways have been proposed to improve the theory [2], beginning with number-projected BCS (PBCS) first proposed by Bayman [3] and Blatt [4]. It is our aim here to evaluate the PBCS by testing it in situations for which an exact solution is available.

To investigate the accuracy of PBCS approximation we shall consider a finite number of spin- $1 / 2$ fermions, e.g., neutrons or protons, distributed in a sequence of singleparticle levels and interacting through a pairing force. We will mainly consider the reduced BCS Hamiltonian given by

$$
H=\sum_{i}^{\Omega} \varepsilon_{i}\left(a_{i}^{\dagger} a_{i}+a_{\bar{i}}^{\dagger} a_{\bar{i}}\right)-g \sum_{i, j}^{\Omega} a_{i}^{\dagger} a_{\bar{i}}^{\dagger} a_{\bar{j}} a_{j} .
$$

Here $g$ is the strength of the pairing force acting in a space of $\Omega$ two-fold degenerate orbitals with the singleparticle energies $\varepsilon_{i}$.

The Hamiltonian (1) is exactly solvable [5, 6] and it was used in 60ies to make a critical analyses of the BCS approximation in finite Fermi systems. Thus in Ref. [6] Richardson studied the exact and the BCS solutions of the Hamiltonian (1) with $\varepsilon_{i}=i$ and for systems with $\Omega=8-32$ at half filling, i.e., with the number of particles $N=\Omega$. Such systems plausibly model the pairing in deformed nuclei with the active nucleons (for pairing calculations) filling the major shells 8-20, 50-82 and 82-126. The main conclusion of Ref. [6] was that BCS model strongly underestimate the pairing correlations even for relatively large values of the pairing strength. The question we address in this study is how much one could improve the BCS results relative to the exact model if we perform PBCS calculations. Some of the issues analyzed in this paper where also discussed recently in relation to metallic grains studies $[8,9,10]$. There has also been a recent study in the nuclear physics context 11]. These authors found a significant difference between the exact solution and the PBCS. In Sec. II we shall argue that the PBCS approximation is nevertheless quite accurate if it is applied in a limited window around the Fermi level of the order of one major shell in atomic nuclei.

Unfortunately, Richardson's model requires that the interaction matrix elements be equal in the pairing Hamiltonian [12]. It is essential to be able to treat the most general form of the Hamiltonian (1), with the matrix elements computed as integrals over an effective twoparticle potential, if the theory is to be a global one describing the entire nuclear mass table. In that case the Hamiltonian has a more general form

$$
H=\sum_{i}^{\Omega} \varepsilon_{i}\left(a_{i}^{\dagger} a_{i}+a_{\bar{i}}^{\dagger} a_{\bar{i}}\right)-\sum_{i>j}^{\Omega} v_{i j} a_{i}^{\dagger} a_{\bar{i}}^{\dagger} a_{\bar{j}} a_{j},
$$

where $v_{i j}=v_{i \bar{i} j \bar{j}}$ are calculated with some two-body interaction such as in Eq.(16) below. There is no algebraic solution for this more general case, but we can obtain useful results up to and beyond $\Omega=16$ using ordinary numerical matrix methods. Some examples will be treated in Sec. III. The realistic calculations show that the PBCS approximation gives accurate results, confirming the conclusions based on Hamiltonian (1) and Richardson model. The fact that PBCS can provide a good description for the ground state of realistic Hamiltonians can be also seen from the large overlaps betweent the PBCS and the shell model wave functions [13].

A good agreement between PBCS and the exact Richardson's solution we also find for the occupation probabilities of single-particle levels, analysed in Sec. IV. It is worth to emphasize that this agreement is obtained with two different wave functions for the ground state 
of the systems, i.e., a condensate formed by identical Cooper pairs in PBCS model, a non-condensate structure based on distinct pairs in the case of exact Richardson's solution. These differences manifest clearly in the collectivity of the Cooper pairs, discussed in Sec. IV. The reason why such differences do not affect significantly the correlation energies and the occupation probabilities when the calculations are done in limited window around the Fermi level is not yet clear [14].

Finally we would like to mention that another methodology that is widely used to go beyond the BCS theory is the Lipkin-Nogami approximation. We do not consider it here for following reasons. First, it has been thoroughly studied in the past and its strengths and deficiencies are well known. It has a serious shortcoming in that the approximation is not reliable near closed shell nuclei [16]. Since we seek approximation methods that cover all the extremes that arise in the nuclear mass table, we find this method unsuitable.

\section{SOLUTIONS OF THE BCS HAMILTONIAN}

The Hamiltonian (1) certainly describes the basic features of nuclear pairing correlations, and it is commonly solved using the BCS or PBCS approximation. Both BCS and PBCS methods are variational in that the approximation is made on the wave function, and the energy is calculated as an expectation value. The usual form of the BCS wave function is the well-known expression $\Pi_{i}^{\Omega}\left(u_{i}+v_{i} a_{i}^{\dagger} a_{i}^{\dagger}\right)|\rangle$, but for putting it in the context of the other treatments one can write it as an exponentiated product of a pair operator,

$$
\Gamma^{\dagger}=\sum_{i} x_{i} a_{i}^{\dagger} a_{\bar{i}}^{\dagger} .
$$

The BCS ground state can then be expressed as a coherent superposition of pairs, i.e.,

$$
|B C S\rangle \propto e^{\Gamma^{\dagger}}|0\rangle \equiv \sum_{n} \frac{\left(\Gamma^{\dagger}\right)^{n}}{n !}|0\rangle .
$$

The mixing amplitudes of the pair operator, written usually as $x_{i}=v_{i} / u_{i}$, are given by the well-known BCS equations. The PBCS approximation is obtained by restricting the expansion in Eq.(3) to the term having the required number of particles. Thus, in PBCS the ground state wave function can be expressed

$$
|P B C S\rangle \propto\left(\Gamma^{\dagger}\right)^{N_{\text {pair }}}|0\rangle,
$$

where $N_{\text {pair }}$ is the number of pairs. The PBCS equations, which determine the mixing amplitudes $x_{i}$ of the pair operator (2), are derived by minimizing the average of the Hamiltonian in the state (5). They can be solved by using the residual integrals technique [15]. Alternatively, if the number of pairs is not too large, the amplitudes $x_{i}$ can be found by using the technique of recurrence relations.
As shown in Ref. [5], the pairing Hamiltonian (1) can be solved exactly. The solution resembles eq. (5) except that the operator $\Gamma^{\dagger}$ is replaced by $N_{\text {pair }}$ different pair operators $B_{\nu}^{\dagger}$,

$$
|\Psi\rangle=\prod_{\nu}^{N} B_{\nu}^{\dagger}|0\rangle .
$$

The pair operators have the form

$$
B_{\nu}^{\dagger}=\sum_{i} \frac{1}{2 \varepsilon_{i}-E_{\nu}} a_{i}^{\dagger} a_{i}^{\dagger} .
$$

They depend on energy parameters $E_{\nu}$ obtained by solving the set of nonlinear equations

$$
\frac{1}{g}-\sum_{j} \frac{1}{2 \varepsilon_{j}-E_{\nu}}+\sum_{\mu \neq \nu} \frac{2}{E_{\mu}-E_{\nu}}=0 .
$$

The sum of pair parameters $E_{\nu}$ gives the total energy of the system, i.e.,

$$
E=\sum_{\nu} E_{\nu}
$$

In the limit $g=0$ the pair energies $E_{\nu}$ of the ground state solution coincide with the lowest single-particle energies, i.e., $E_{\nu}=2 \varepsilon_{\nu},\left(\nu=1,2, \ldots N_{\text {pair }}\right)$. When the interaction is turned on, the pair energies evolve toward lower values and could become complex two at a time. This fact was used by Richardson to obtain a set of equations in which the singularities are removed [6].

For small values of $g$ in finite systems, the BCS condensate collapses and the BCS approximation gives zero correlation energy. On the other hand, due to the finite distance between the levels, for small values of the interaction strength the pairing interaction energy can be calculated perturbatively. This is opposite to what happens in finite systems where the pairing correlations depends exponentially on pairing strength in the weak coupling limit. The perturbative solution can be easily derived by enumerating the two-particle two-hole configurations starting from the lowest energy configuration, or by taking the small- $g$ limit of the Richardson equations [9].

The second-order perturbation result for the interaction energy, i.e., the energy gained by the system when the interaction is turned on, is given by

$$
E_{\text {corr }}^{P}=\frac{g^{2}}{2} \sum_{i=1}^{N_{\text {pair }}} \sum_{j=N_{\text {pair }}+1}^{\Omega} \frac{1}{\varepsilon_{j}-\varepsilon_{i}}
$$

This expression is valid for even $N$; for odd $N=N_{\text {pair }}+1$ the $j$ sum begins at $j=N_{\text {pair }}+2$. It can be shown [9] that the approximation (10) for the interaction energy is valid if $g<g_{P} \equiv g^{*}\left(1-g^{*}\right)$, where $g^{*}$ is the convergence radius of the perturbative expansion given by

$$
\frac{1}{g^{*}}=\sum_{j=1}^{N_{\text {pair }}} \frac{1}{\varepsilon_{j}}
$$



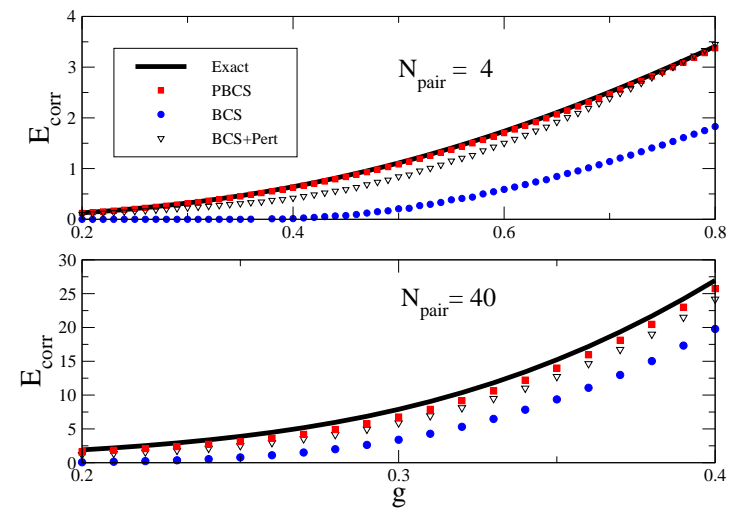

FIG. 1: Correlation energies for the Hamiltonian (1), calculated in various approximations. Upper panel: $\Omega=8, N_{\text {pair }}=$ 4; lower panel: $\Omega=80, N_{\text {pair }}=40$.

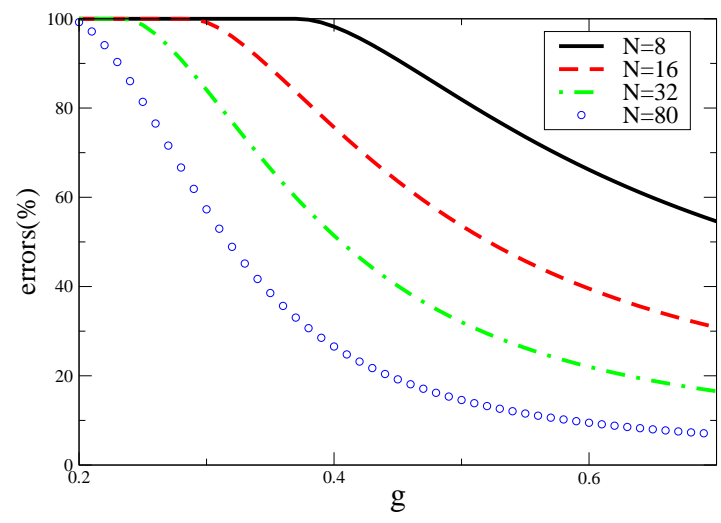

FIG. 2: Errors of the BCS approximation for correlation energies.

\section{A. Correlation Energies and Pairing Gaps}

In this section we shall discuss the correlations energies and the pairing gaps provided by the Hamiltonian (1) for a single-particle spectrum formed by $\Omega$ two-fold degenerate orbits with uniformly spaced energies, i.e., $\varepsilon_{i}=i$. The analyses is done for systems formed by $\mathrm{N}$ particles distributed in $\Omega=N$ levels with $\Omega=8-80$. For the strength of the interaction we use the range $g=0.1-0.8$, which covers all the interesting coupling regimes met in nonspherical atomic nuclei. In all figures discussed in this section the energies, the pairing gaps and the interaction strength are given in units of single-particle levels spacing.

We first discuss the correlation energies calculated ex-

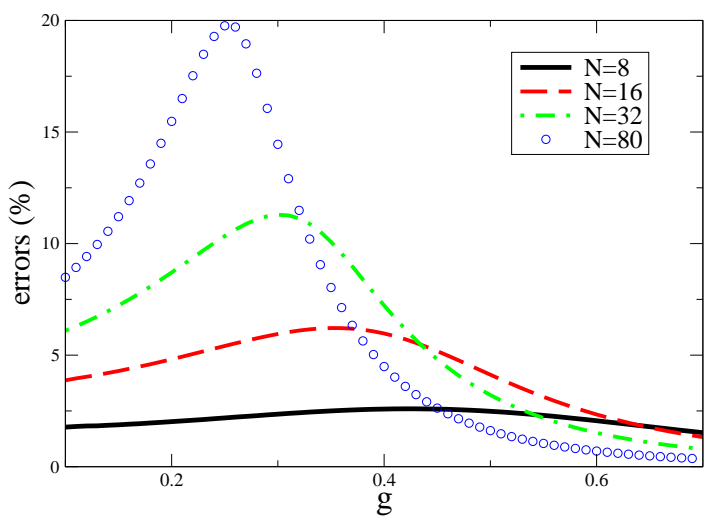

FIG. 3: Errors for the correlation energies calculated in the PBCS approximation.

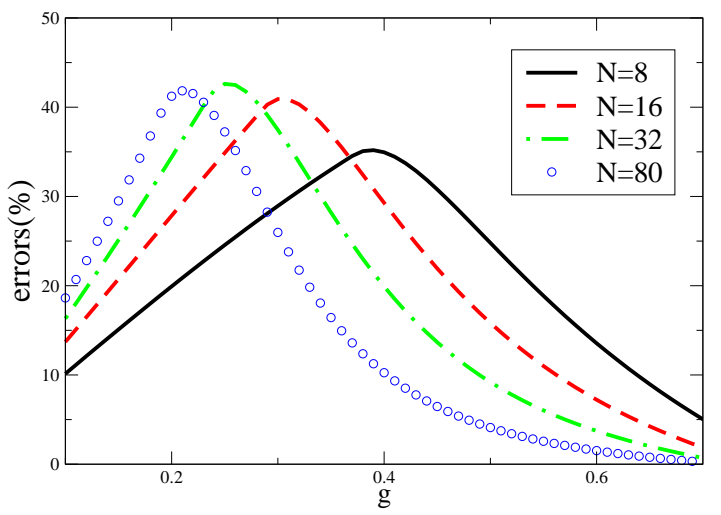

FIG. 4: Errors for the correlation energies approximated as the sum of the BCS energy and the second-order perturbative energy, Eq. (13).

actly and in various approximations. The correlation energies are defined by the difference in energies between a single Slater determinant and the pair-correlated state, i.e.,

$$
E_{c o r r}(g)=E_{H F}-E(g) \text {. }
$$

The results are shown in Fig.1. From this figure is obvious that the BCS seriously underpredicts the correlation energy while the PBCS does much better. To see the differences more quantitatively, in the next figures we show for each approximation how the error depends on $g$ and $\Omega$. The BCS errors shown in Fig. 2 are large, making this approximation completely unreliable. The results for the PBCS approximation are shown in Fig. 3. The errors are much smaller, but become unacceptably large for the 
biggest space, $\Omega=80$. In spacing corresponding to a single major shell the error is well under $10 \%$. It is important to emphasize that the example discussed above does not contradict the fact that pairing models become more accurate if one moves toward the thermodynamical limit [7]. To reach this limit the particle number is increased but, in the same time, the calculations are done keeping a fixed energy window around the Fermi energy. In this way the increase of particle number has as effect an increase of level density around the Fermi level which, in turn, is increasing the pairing correlations and by that the accuracy of pairing models. This is different from what happens in the system with 40 pairs discussed above and in many BCS and HFB calculations performed for atomic nuclei, where the energy window around the Fermi level is increased such that to include the deep bound nucleons. As discussed above, by this procedure the accuracy of pairing calculations becomes worse not better.

The question which arises is why BCS approximation strongly underestimates the pairing correlations in finite systems. This question was recently addressed in relation to metallic grains calculations. Thus, in Ref. 9 ] it is argued that BCS works properly only for so-called "condensed" levels, i.e., the levels with the energies located in the interval $I=|\Delta-\mu|$, where $\Delta$ is the gap parameter in the BCS equations and $\mu$ is the Fermi energy. This conclusion is supported by the observation that the correlation energy calculation in BCS is close to the exact result obtained if from the exact solution is kept only the contribution of condensed levels (in the exact solution the condensed levels have usually complex pair energies). On the other hand, it was found that the contribution of levels located outside the interval $I$ are underestimated in BCS. Since in the exact solution the Cooper pairs corresponding to these levels have the pair energies close to the single-particle levels, one expects that the contribution of these levels to pairing correlations could be treated perturbatively. Based on these observations it was found [8, 9, 10] that in metallic grains the correlation energy could be approximated by a formula combining the BCS and the perturbative expressions in the sum $E_{\text {corr }}^{B C S}(g)+\Delta+a(g) E_{\text {corr }}^{P}$, where the last term is the perturbative result and $a(g)$ is a function of the order of unity determined by the fitting protocol. The BCS contribution is approximated by the first two terms representing the condensation energy and the pairing gap for infinite system. The later accounts for the size correction to the bulk result (first term). In order to see if such an approximation could work for the small systems analyzed here, we have simply replaced the first two terms in the above expression by the BCS result (for finite systems) and we take $a(g)=1$,

$$
E_{\text {corr }}=E_{\text {corr }}^{B C S}+E_{\text {corr }}^{P} \text {. }
$$

The corresponding results are shown in Fig. 1 by inverted triangles and the corresponding errors are given in Fig. 4. It can be seen that this simple approximation

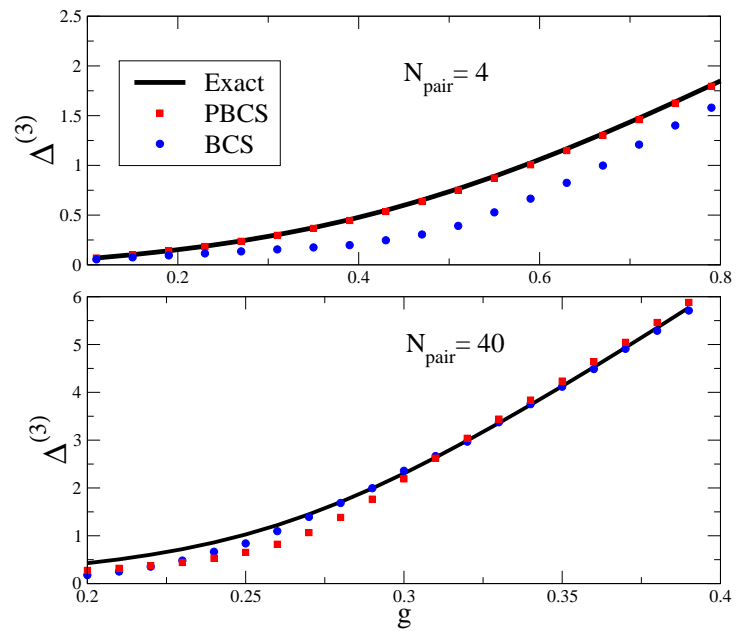

FIG. 5: Pairing gaps (Eq. (14) ) calculated in various approximations. Upper panel: $\Omega=8, N_{\text {pair }}=4$; lower panel: $\Omega=80, N_{\text {pair }}=40$.

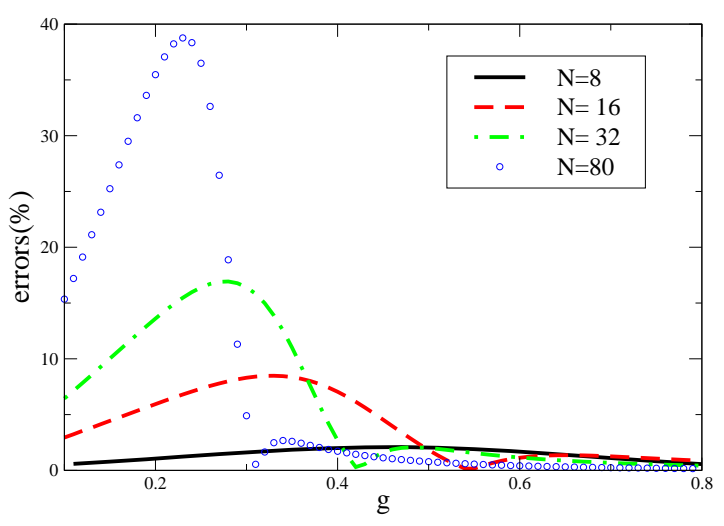

FIG. 6: Error in pairing gaps calculated with the PBCS approximation.

works surprisingly well for a wide range of the pairing strengths, including the physical region up to $g \sim 0.8$. Of course, it is not applicable to situations where there is a degeneracy in the single energies at the Fermi level, since perturbation theory diverges in that situation.

We next compare the pairing gaps calculated in the BCS and PBCS approximations with the exact values. The gap is defined as the second difference of energies between an odd- $N$ system and its neighbors with even$N$,

$$
\Delta^{(3)}(N)=\frac{1}{2}(2 E(N)-E(N-1)-E(N+1)) .
$$

We shall call $\Delta^{(3)}(N)$ the gap at number $N$. The results at the smallest and largest spaces are shown in Fig. 5. 
One can see that PBCS gives accurate gaps in all coupling regimes, contrary to the larger systems analyzed in metallic grain studies [8, 9, 10]. The errors associated with the PBCS approximation are shown in more detail in Fig. 6. It is probably acceptable to tolerate an error up to $10 \%$ for the gap, but not higher. This confirms for the gaps that the PBCS is only reliable up to moderate size spaces.

It is interesting to note that in spite of the large errors in the correlation energies, in the physical region of the strength parameter the BCS pairing gaps come much closer to the exact results. However, contrary to the PBCS results, the good agreement of the BCS gaps to the exact values in the region of well-developed pairing correlations is just a manifestation of the errors cancellation when the subtraction is performed in equation (14). Consequently, fixing the pairing force by the odd-even mass difference, as usually done in nuclear structure calculations, does not guarantee a good description of correlation energies within the BCS approximation.

\section{PBCS FOR REALISTIC PAIRING INTERACTIONS}

Ultimately, the theory of nuclear binding energies should be based on realistic interactions dropping the constant- $g$ approximation. The Richardson solution has been somewhat generalized to encompass separable pairing interactions [26], but to be truly realistic the Hamiltonian must allow completely general interactions in the many-body space of pairwise occupated orbitals. These Hamiltonians can be solved by straightforward configuration interaction (CI) methodology, in which a Hamiltonian matrix is constructed in the Fock space of the orbitals and diagonalized by standard linear algebra operations. The size of the space $D$ needed to represent the most general paired wave function is given by the number of combinations of $N_{\text {pair }}$ orbitals out of total of $\Omega$,

$$
D=\left(\begin{array}{l}
\Omega \\
N_{\text {pair }}
\end{array}\right) \text {. }
$$

For example, for $\Omega=16$ orbitals and $N=16$ particles the dimension of the space is 12,870 . The lowest eigenvector for a space of this size is easily calculated on serial computers using the Lanczos algorithm.

We have carried this out for two examples in which the pairing is very different. The Hamiltonian makes use of the orbital energies and wave functions from the global mean-field calculations of Ref. [17], which are based on the Skyrme SLy4 energy functional. Pairing is strong in the Sn isotope chain, and we will take ${ }_{67}^{117} \mathrm{Sn}_{50}$ and its neighbors as an example of where the pairing is well developed. The second example is ${ }^{207} \mathrm{~Pb}$. In the global systematics of neutron pairing gaps, the one at ${ }^{207} \mathrm{~Pb}$ is the smallest $\left(\Delta^{(3)}=0.32 \mathrm{MeV}\right)$, so this provides a good test of the approximation methods in the weak pairing limit.
We use the published code ev8 [18] to recalculate the needed orbital properties, starting from the wave functions provided in the original global survey [17]. The orbitals are represented internally in the code with a 3dimensional mesh, so they need not have good angular momentum quantum numbers. However, for the even- $N$ $\mathrm{Sn}$ and $\mathrm{Pb}$ isotopes, the mean field solution is spherically symmetric and the orbitals can be given angular momentum assignments. In Ref. [17] the pairing interaction was taken as a density-dependent contact interaction in a space truncated to a band of width $10 \mathrm{MeV}$ about the Fermi energy. Here we use an ordinary delta function to generate the pairing matrix elements,

$$
v_{i j}=v_{0} \int d^{3} r\left|\phi_{i}(r)\right|^{2}\left|\phi_{j}(r)\right|^{2},
$$

where the $\phi(r)$ are orbital wave functions. The strength $v_{0}$ has been fitted to the global systematics of pairing gaps [19] and the self-consistent orbitals were generated with that value. The single-particle energies and the matrix of $v_{i j}$ values were then used as input data for separate codes to solve the Hamiltonian Eq. (2). We calculate the correlation energies and the pairing gaps using a range of values for $v_{0}$ obtained by scaling the matrix elements obtained from the ev8 code.

For the Sn isotopes with neutron number $N$ around 68 , there are $\Omega=16$ orbitals in the $10 \mathrm{MeV}$ window, originating from the $d_{5 / 2}, g_{7 / 2}, s_{1 / 2}, d_{3 / 2}$ and $h_{11 / 2}$ shells of the spherical shell model. This space is small enough to permit the exact calculations to be performed without special numerical difficulties. Fig. 7 shows the neutron correlation energy in ${ }^{116} \mathrm{Sn}$ as a function of interaction strength $v_{0}$. The range for $v_{0}$ includes the value $v_{0} \sim 450 \mathrm{MeV} \mathrm{fm}^{3}$ that has been fitted to the global gap systematics using the BCS approximation [19]. The comparison in Fig. 7 confirms the results obtained with the Hamiltonian with the constant- $g$ pairing. Namely, the BCS systematically underpredicts the correlation energy while the PBCS tracks the exact energy very well. PBCS describes also very well the pairing gaps, as shown in Fig. 8. There is also a good agreement for the BCS gaps but, as already noticed in the previous section, this agreement is in fact a manifestation of errors cancelation.

Next we show the results for $\mathrm{Pb}$. Here the energy truncation to a $10 \mathrm{MeV}$ window gives a space that is still too large to easily perform the exact calculation, so we truncated it further $(\sim 8 \mathrm{MeV}$ window) to leave $\Omega=16$ orbitals. Fig. 9 shows the correlation energy in this space and the BCS and PBCS approximations. One sees that the PBCS keeps an accuracy much better than $100 \mathrm{keV}$, while the BCS is off by more than a half of an MeV. The correlation energies in the other nuclei needed for the ${ }^{207} \mathrm{~Pb}$ gap are very small and in BCS there is no condensate in ${ }^{207} \mathrm{~Pb}$ and ${ }^{208} \mathrm{~Pb}$. As a result, the BCS error for the ${ }^{206} \mathrm{~Pb}$ correlation energy is not well canceled in formula for the gap energy. Fig. [10] shows the calculated gap in the three treatments. One sees again that the 


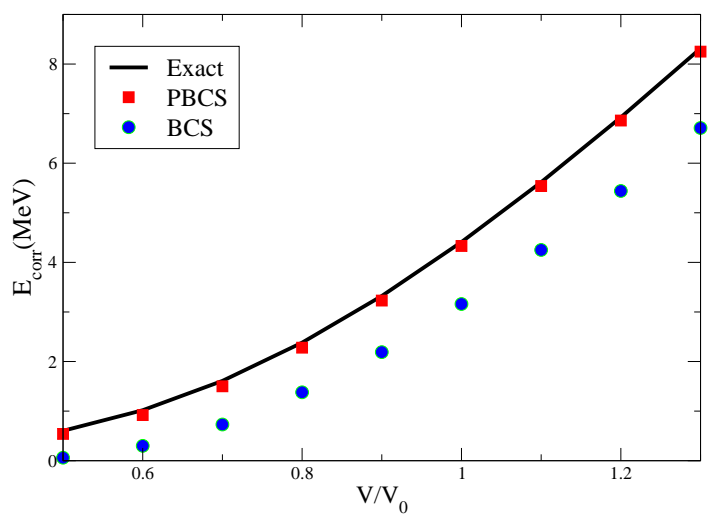

FIG. 7: Pairing correlation energy in ${ }^{116} \mathrm{Sn}$. The pairing interaction is a delta function with a strength scaled from the nominal value $v_{0}=465 \mathrm{MeV}-\mathrm{fm}^{3}$ by a factor given on the abscissa. The orbital space is the 16 orbitals around the Fermi energy as described in the text. Solid line shows the result of exact diagonalization.

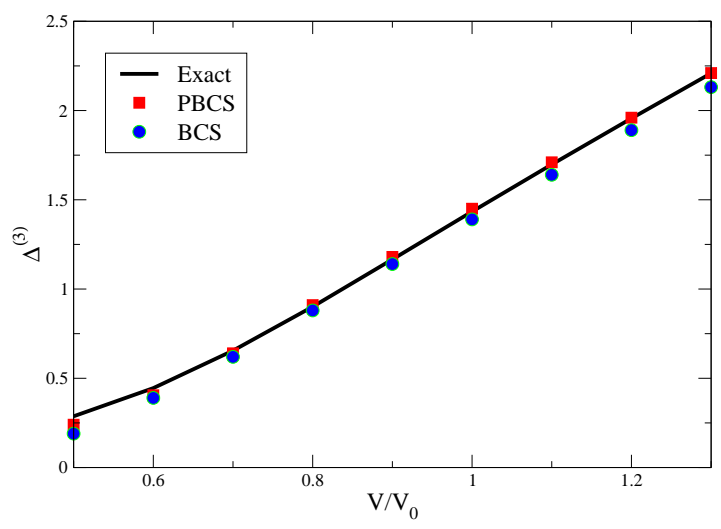

FIG. 8: Neutron pairing gap at ${ }^{117} \mathrm{Sn}$. The correlations energies for the three nuclei needed for Eq. (14) were calculated with the same functional and in the same space as in Fig. 7.

PBCS is remarkably accurate. The BCS error of $100-200$ $\mathrm{keV}$ is quite significant on the scale of the empirical gap energies, which fluctuate around an average of $1 \mathrm{MeV}$ with an r.m.s. deviation of $300 \mathrm{keV}$.

\section{OCCUPATION PROBABILITIES AND TWO-BODY CORRELATIONS}

To probe the accuracy of BCS-based models relative to the exact solution we have also analyzed the occupation probabilities and the two-body correlations induced by

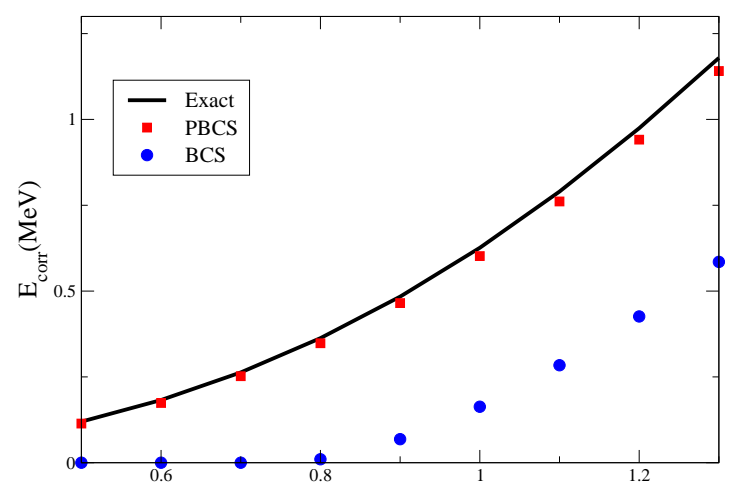

FIG. 9: Pairing correlation energy in ${ }^{206} \mathrm{~Pb}$. For these calculations, the space was truncated to $\Omega=16$ by including the 11 highest orbitals below the $N=126$ magic number and the $5 g_{9 / 2}$ orbitals above $N=126$. This corresponds to an energy window of about $8 \mathrm{MeV}$.

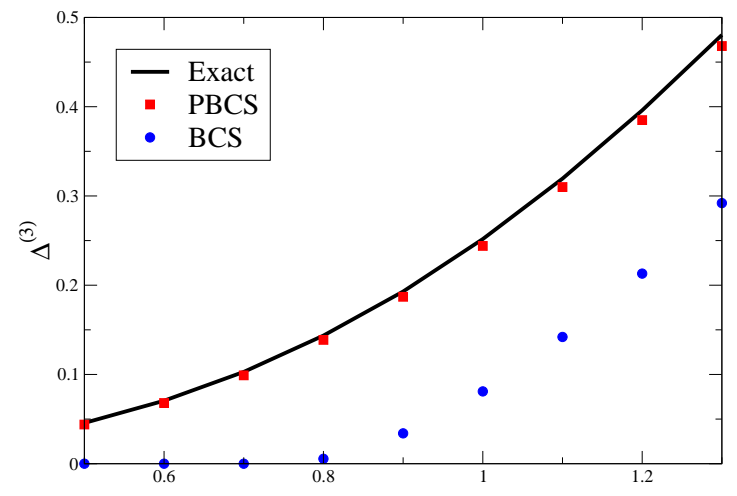

FIG. 10: Neutron pairing gap at ${ }^{207} \mathrm{~Pb}$. For these calculations we used the same space as in Fig. 9.

the pairing force. The results discussed in this section correspond to the Hamiltonian (1) with $\varepsilon_{i}=i$.

\section{A. Occupation probabilities}

The BCS occupation probabilities are calculated by solving the standard BCS equations (here we take into account the renormalisation of the single-particle energies by the diagonal term of the interaction) while the PBCS values are obtained by using the residual integral method described in Ref. [15].

In the exact solution the occupation probabilities $v_{i}^{2}$ 


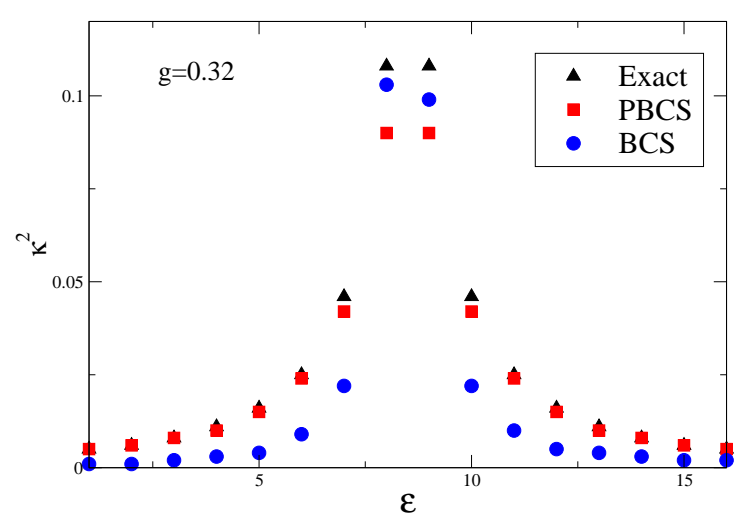

FIG. 11: Occupation factors $\kappa_{i}^{2}=v_{i}^{2}\left(1-v_{i}^{2}\right)$ for $N_{\text {pair }}=8$, $\Omega=16$ and $g=0.32$. The results correspond to the Hamiltonian (1) with $\varepsilon_{i}=i$.

are given by [ $[6]$

$$
v_{i}^{2}=\sum_{\nu=1}^{N} \frac{a_{\nu}}{\left(2 i-E_{\nu}\right)^{2}},
$$

where $a_{\nu}$ are obtained by solving the set of equations

$$
\left[C_{\nu}-2 \sum_{\mu} \frac{1}{\left(E_{\mu}-E_{\nu}\right)^{2}}\right] a_{\nu}+2 \sum_{\mu} \frac{a_{\mu}}{\left(E_{\mu}-E_{\nu}\right)^{2}}=1
$$

and $C_{\nu}$ are given by

$$
C_{\nu}=\sum_{i=1}^{2 N} \frac{1}{\left(2 i-E_{\nu}\right)^{2}}
$$

To solve the equations above is convenient to rewrite them in terms of the real and the imaginary parts of pair energies $E_{\nu}$. The corresponding expressions can be found in Ref. [6].

For the discussions below we shall use the product between the occupation and non-occupation probabilities, i.e., $\kappa_{i}^{2} \equiv v_{i}^{2}\left(1-v_{i}^{2}\right)$, which provides relevant informations on the diffusivity of the Fermi sea and the entanglement properties of pairing tensor (see Eq.(21) below).

In Figs. 11-13 are shown the values of $\kappa_{i}^{2}$ for a system with $\mathrm{N}=8$ pairs and for three values of the strength parameter corresponding to the weak $(g=0.32)$, intermediate $(g=0.42)$ and strong coupling $(g=0.87)$ regimes. For these strength values the gaps (exact results) are approximatively equal to $0.6,1.0,5.0$, respectively. As seen from Figs. 11-13, the values of $\kappa_{i}^{2}$ for the states outside the interval $I=|\Delta-\mu|$ are rather well described by PBCS and underestimated by BCS. On the other hand, one can notice that for the states which are the closest to the chemical potential BCS gives in the weak and intermediate coupling regimes larger values than PBCS

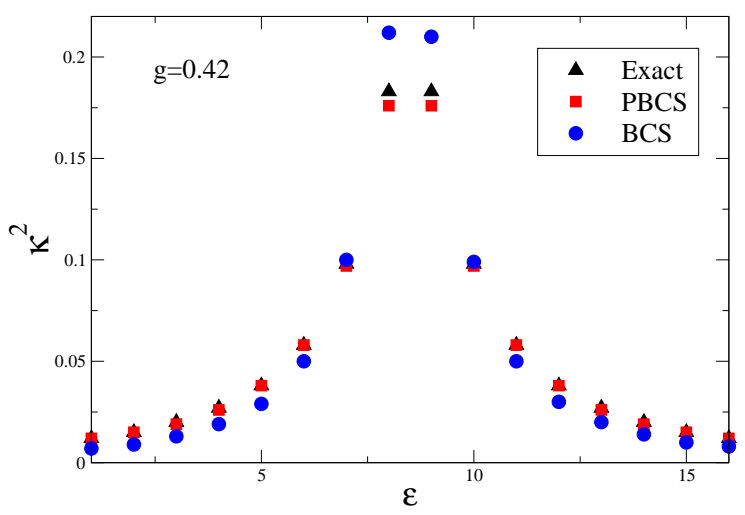

FIG. 12: The same as in Fig. 11 but for $g=0.42$

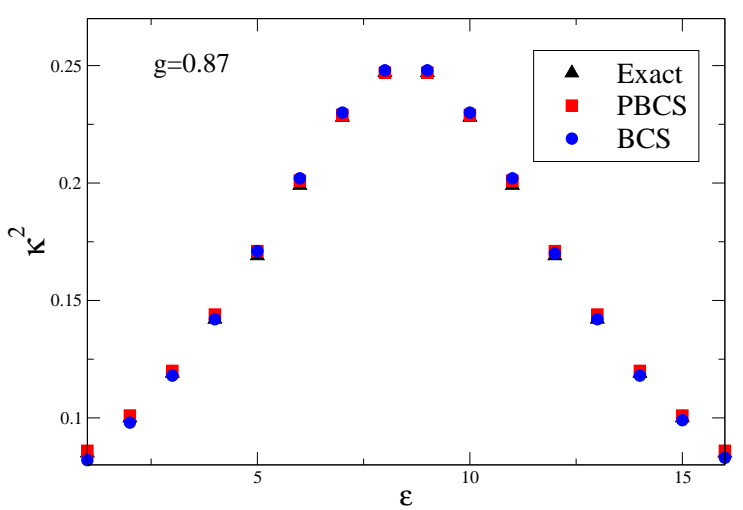

FIG. 13: The same as in Fig. 11 but for $g=0.87$

and the exact solution. In the strong coupling regime shown in Fig. 13, when all levels are inside the interval $I$, the BCS and PBCS results become close to the exact values for all levels included in the calculations. Hence, when the pairing correlations are well-developed, BCS describes rather well the occupation probabilities even though the errors for the condensation energies are large.

\section{B. Two-body correlations}

The distribution of occupation probabilities around the Fermi level determines the intensity of two-body correlations, which could be eventually probed in pair transfer processes. Two-body correlations are commonly intro- 
duced by the two-body density defined by

$$
\begin{aligned}
\rho_{2}\left(x_{1}, x_{2}\right) & =\sum_{\sigma_{3} . . \sigma_{N}} \int\left|\Psi\left(x_{1}, x_{2}, \ldots, x_{N}\right)\right|^{2} d \vec{r}_{3} \ldots d \vec{r}_{N} \\
& =\left\langle 0\left|a^{+}\left(x_{1}\right) a^{+}\left(x_{2}\right) a\left(x_{2}\right) a\left(x_{1}\right)\right| 0\right\rangle,
\end{aligned}
$$

where $\Psi$ is the many-body wave function, $x$ denotes the radial and spin coordinate, i.e., $x \equiv \vec{r} \sigma$, and $a^{\dagger}(x)$ is the particle creation operator. To separate the genuine twobody correlations induced by pairing correlations from the correlations of Hartree-Fock type, the two-body density is usually written in the following form [20]

$$
\rho_{2}\left(x_{1}, x_{2}\right)=\rho\left(x_{1}\right) \rho\left(x_{2}\right)-\left|\rho\left(x_{1}, x_{2}\right)\right|^{2}+\left|\kappa\left(x_{1}, x_{2}\right)\right|^{2},
$$

where $\rho(x)$ is the (local) particle density while $\rho\left(x_{1}, x_{2}\right)$ is the non-local (exchange) part of particle density. The last term defines the genuine two-body correlations, i.e., the correlations not included in the independent mean-field picture of fermion motion. In the BCS approximation the last term corresponds to the pairing tensor in the coordinate representation, i.e.,

$$
\kappa\left(x_{1}, x_{2}\right)=\left\langle 0\left|a\left(x_{2}\right) a\left(x_{1}\right)\right| 0\right\rangle=\sum_{i} \kappa_{i} \varphi_{i}\left(x_{1}\right) \varphi_{\bar{i}}\left(x_{2}\right),
$$

where $\kappa_{i} \equiv\left\langle 0\left|a_{i} a_{\bar{i}}\right| 0\right\rangle=u_{i} v_{i}$ is the pairing tensor in a single-particle basis defined by the operators $a_{i}^{\dagger}$ and $\varphi_{i}$ are the associated wave functions.

According to its definition, the pairing tensor in the coordinate representation provides information about the spatial correlations between two nucleons irrespective if they belong or not to the same Cooper pair. The spatial structure of these correlations in atomic nuclei have been recently discussed in Refs. [21, 22]. If we need to investigate only the spatial correlations between the nucleons belonging to the same pair, instead of pairing tensor one should analyse the pair wave function. The latter has a different structure in BCS-based models compared to the exact solution. Thus, in BCS and PBCS models all pairs are described by the same wave function

$$
\phi\left(x_{1}, x_{2}\right)=C \sum_{i} x_{i} \varphi_{i}\left(x_{1}\right) \varphi_{\bar{i}}\left(x_{2}\right),
$$

where the mixing amplitudes $x_{i}$ and the normalization factor $C$ depend on the approximation used to describe the condensate. Thus, in BCS $x_{i}=v_{i} / u_{i}$ and $C=$ $\sum_{i} v_{i}^{2} / u^{2}$, where $v_{i}, u_{i}$ are the occupation amplitudes provided by the BCS equations. Formally, the same expressions can be used for the PBCS model but in this case the amplitudes $v_{i}, u_{i}$ are just variational parameters, not occupation amplitudes.

For the exact solution each pair is described by a specific wave function, i.e.,

$$
\phi_{\nu}\left(x_{1}, x_{2}\right)=\sum_{i} \frac{1}{2 \varepsilon_{i}-E_{\nu}} \varphi_{i}\left(x_{1}\right) \varphi_{\bar{i}}\left(x_{2}\right) .
$$

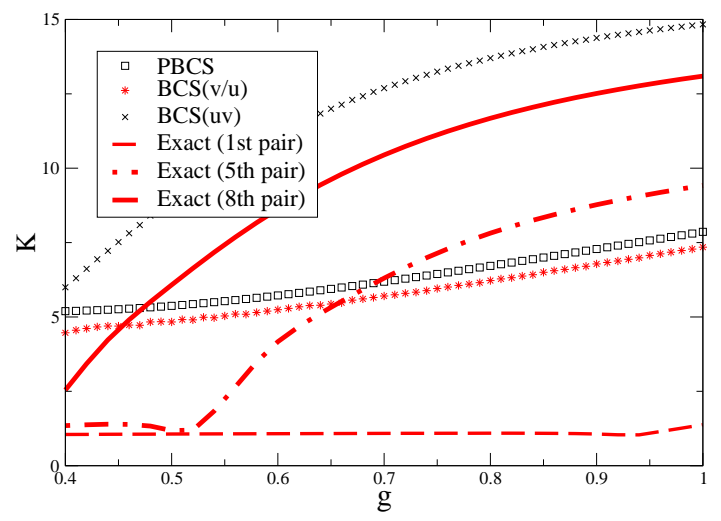

FIG. 14: The Schmidt numbers (Eq. 24) for a system with $N_{\text {pair }}=8$ and $\Omega=16$.

Therefore the correlations among the nucleons belonging to the same pair can be rather different according to the pair function they belong to. To characterize the amount of correlations in the pairing tensor (21) and in the pair wave functions (22-23) we employ the quantity [23]

$$
K=1 / \sum_{i} w_{i}^{4}
$$

where $w_{i}$ are the mixing amplitudes of the normalized two-body wave functions (21-23). The quantity $\mathrm{K}$ is sometimes called the Schmidt number [24] and it gives a global indication of the degree of entanglement in twobody systems. Thus, the minimum value of $K$ is 1 and is obtained when in the expansion (24) only one term is non-zero; this case corresponds to no entanglement since the two-body wave function is split in the individual wave functions of the two- particle system. The maximum possible value of $\mathrm{K}$ is obtained when the weights $w_{i}$ have the same value for all terms in the expansion.

In Fig 14 is shown how K number evolves as a function of the strength parameter. One can see that the entanglement properties of Cooper wave functions are rather similar in BCS and PBCS approximations and very different from the entanglement of the correlation function (21). As already stressed above, the latter describes the correlations between two generic fermions, which do not necessarily belong to the same Cooper pair.

A particular behavior have the Cooper pairs (23) described by the exact solution. Their entanglement properties depends on how far are their pair energies $E_{\nu}$ (more precisely, their real part) from Fermi level. Thus, as seen in Fig 14, the 8th pair (i.e., the one corresponding to $E_{8}$ ), which is the closest to Fermi level, is the most entangled. At the other extreme is the 1st pair, corresponding to $E_{1}$, which remains almost uncorrelated in all coupling regimes. An intermediate behavior has the $5 t h$ pair which 
remains uncorrelated up to $g \approx 0.53$ and then its entanglement is increasing rather fast, in a similar fashion as for the $8 t h$ pair. The strength value for which $\mathrm{K}$ number of the 5th pair starts to increase corresponds to the value at which the pair energy $E_{5}$ becomes complex. As mentioned previously, the pair energies become complex when the corresponding level (in the limit $g=0$ ) becomes condensed, i.e., enters in the interval $I=|\Delta-\mu|$. Hence, in the exact solution at a given value of the strength only some pairs become entangled, namely the ones corresponding to the condensed levels. This is very different from what happens in BCS and PBCS models in which all Cooper pairs are identical and therefore all of them have the same entanglement properties.

\section{SUMMARY AND CONCLUSIONS}

In this paper we have studied how reliable are the approximations used to solve the pairing Hamiltonian when the parameters are chosen in a range appropriate to calculate nuclear ground states. As is well known, the BCS approximation does not do well for correlation energies. On the hand, we find that PBCS, with its variation after number projection, is highly accurate over most the interesting parameter range for moderate size orbital spaces. This applies to orbital spaces such as a single major shell or energies truncations of the order of $5 \mathrm{MeV}$ around the Fermi level. Much larger spaces, for example including all the occupied orbitals, give a degradation in the performance of the PBCS that we do not yet understand. However, by renormalizing the pairing interaction, the calculations in large spaces could be reduced to smaller ones in which the PBCS gives better results. One way the renormalization could be done is to demand the same computed gaps when the number of states available for the active pairs is changed. For example, as seen in Fig. 3 , if in the PBCS calculations we decrease the number of active pairs from 40 to 8 and, in order to keep the same gap, we increase the strength from 0.27 to 0.5 , the error in the correlation energy drops from $20 \%$ to below $5 \%$. Hence, to reduce the errors of PBCS calculations is preferable to restrict the calculations to a small number of active particles with energies located close to the
Fermi level. In any case, single-major shell truncations are widely used and there is no reason to not use the PBCS with those conditions.

Both the BCS and PBCS seem to do well on calculating pairing gaps with the the reduced BCS Hamiltonian Eq. (1). However, in the case of BCS the improvement arises from a cancellation of errors. The BCS underpredicts the correlation energies, but more seriously is missing the true values for odd systems. In the realistic case of ${ }^{207} \mathrm{~Pb}$ the errors did not cancel well, and so we regard the BCS as unreliable at the level of $100 \mathrm{keV}$ or so. The PBCS maintained its accuracy for the two realistic cases we considered, confirming our overall assessment of its reliability.

We have also found that PBCS describes accurately the occupation probabilities of the single-particle levels. However, contrary to the agreement found for the correlations energies and the occupation probabilities, the entanglement properties of pair wave functions are very different in PBCS and in the exact solution. Thus, while in PBCS the entanglement of Cooper pairs depends smoothly on the interaction strength, in the exact wave function, formed by non-identical pairs, the entanglement properties of Cooper pairs depend strongly on the position of their pair energies relative to chemical potential.

Finally, we would like to mention that a ground state based on non-identical pairs, specific to the exact solution, could be more appropriate for the description of loosely bound systems such as nuclei close to the drip lines. For such nuclei one expects that the properties of Cooper pairs formed by the valence nucleons moving in loosely bound and continuum single-particle state to be rather different from the pairs formed by the deeper bound nucleons.

\section{Acknowledgments}

We thank Jorge Dukelsky and Stuart Pittel for valuable discussions and for their assistance in solving the Richardson and PBCS equations. This work was supported by the UNEDF SciDAC Collaboration under DOE grant DE-FC02-07ER41457, by DE-FG0200ER41132 and by the grant IDEI nr. 772 .
[1] A. Bohr, B. Mottelson, D. Pines, Phys. Rev. 110 (1958) 936

[2] P. Ring, P. Schuck, The Nuclear Many-Body Problem (Springer-Verlag, 1981)

[3] B. F. Bayman, Nucl Phys 15 (1960) 33

[4] M. Blatt, Progr. Theor. Phys. (Kyoto) 24 (1960) 851

[5] R. W. Richardson, N. Sherman, Nucl. Phys. 52 (1964) 221

[6] R. W. Richardson, Phys. Rev. 141 (1966) 949

[7] R. W. Richardson, J of Math Phys 6 (1965) 1034

[8] J. Dukelsky and G Sierra, Phys Rev Lett 83 (1999) 172;
Phys Rev B61 (2000) 12302

[9] M. Schechter, Y. Imry, Y. Levinson, J. von Deft, Phys. Rev. B63 (2001) 214518

[10] J. von Delft and D. C. Ralph, Physics Reports 345 (2001) 61

[11] G.G. Dussel, S. Pittel, J. Dukelsky, P. Sarriguren, Phys. Rev. C76 (2007)011302(R)

[12] This requirement can be relaxed under certain conditions. See refs. [25], [26] and [27].

[13] N. Sandulescu, J. Blomqvist, T. Engeland, M. HjorthJensen, A. Holt, R. J. Liotta, E. Osnes, Phys. Rev. C55 
(1997) 2708

[14] M. Sambataro, Phys Rev C75 (2007) 054314

[15] K. Dietrich, H J Mang, J. H. Pradal Phys Rev 135 (1964) B22

[16] J. Dobaczewshi and W. Nazarewicz, Phys. Rev. C47 2418 (1993).

[17] M. Bender, G.F. Bertsch, and P-h. Heenen, Phys. Rev. C73 034322 (2006).

[18] P. Bonche, H. Flocard, and P.-H. Heenen, Comput. Phys. Commun. 17149 (2005).

[19] G.F. Bertsch, C. Bertulani, W. Nazarewicz, N. Schunck, and M. Stoitsov, to be published.

[20] C. N. Yang, Rev. of Mod. Phys. 34 (1962) 694
[21] N. Sandulescu, P. Schuck, X. Vinas, Phys Rev C71 (2005) 054303

[22] N Pillet, N. Sandulescu, P. Schuck, Phys. Rev. C76 (2007) 024310

[23] R. Grobe, K. Rzazewski and J. H. Eberly, J. Phys. B27 (1994) L503

[24] C. K. Law, Phys. Rev. A71 (2005) 034306

[25] J. Dukelsky, S. Pittel, Rev. Mod. Phys. 76 (2004) 643

[26] A.B. Balantekin and Y. Pehlivan, Phys. Rev. C76 051001 (2007).

[27] F. Pan, J.P. Draayer, and W.E. Ormand, Phys. Lett. B422 1 (1998). 